\title{
ALTERACIONES DEL SENTIDO DEL GUSTO Y DEL OLFATO CON EL TRATAMIENTO QUIMIOTERÁPICO
}

\section{CHEMOTHERAPY-RELATED TASTE AND SMELL DISORDERS}

Raquel Molina-Villaverde

Servicio de Oncología Médica. Hospital Universitario Príncipe de Asturias.

Resumen

El tratamiento con quimioterapia puede producir muchos efectos adversos. Aunque no sea un efecto secundario muy estudiado ni al que se le haya prestado mucha atención, uno de los más comunes es la alteración del sentido del gusto y/o del olfato secundarios a citostáticos como el cisplatino, los taxanos y la ciclofosfamida. Hasta un $75 \%$ de los enfermos que reciben quimioterapia presentan estas alteraciones y se ha demostrado que altera la calidad de vida de los pacientes. En 1999, estos trastornos fueron incorporados en la escala de toxicidad del Instituto Nacional del Cáncer.

Hay una necesidad urgente de prestar una mayor atención a este efecto secundario con una correcta información a los pacientes y un mayor número de estudios que lo investiguen para introducir mejoras en su manejo. vida.

Palabras clave: Gusto; olfato; calidad de

\section{PLANTEAMIENTO DEL PROBLEMA}

El sentido del gusto y/o del olfato puede cambiar durante el tratamiento oncológico específico.

Para poder comprender mejor este problema, conviene hacer un breve resumen

\section{Abstract}

Chemotherapy agents can cause a wide range of adverse effects. Taste related symptoms are a common toxicity of the chemotherapic agents like cisplatin, taxanes or cyclophosphamide. Although the loss of olfactory and gustatory function is not outwardly apparent, it affects the patient substantially in daily life. Subjective taste and smell changes is in $75 \%$ of cancer patients undergoing chemotherapy. Taste alterations have been incorporated in the National Cancer Institute Common Toxicity Criteria since 1999. There is evidence that this type of alterations in cancer patients negatively affect quality of life. It is very important to inform the patient about this adverse effect because the recovery process will be slow and may take many months. There is an urgent need for increased attention to this effect, both in research and in clinical practice.

Keywords: Taste; olfaction; quality of life.

\section{Correspondencia:}


la información al cerebro a través de los nervios facial, glosofaríngeo y vago. Hay zonas específicas de la lengua que perciben el sabor dulce, el ácido, el salado y el amargo de los alimentos.

En general, los trastornos del sentido del gusto se producen por afecciones que interfieren en el acceso de las sustancias a las células de las papilas gustativas, por trastornos que lesionan estas células receptoras (pérdida sensorial) o por lesión de los nervios aferentes y de las vías gustativas centrales $^{(1)}$.

La causa más frecuente de disfunción del sentido del gusto en la práctica clínica son los efectos adversos de distintos fármacos.

Podemos distinguir varios tipos de trastornos relacionados con la percepción del sabor. La ageusia es la ausencia de la percepción de uno o más sabores básicos (salado, dulce, amargo o ácido); la hipogeusia es la disminución de la sensibilidad frente a algunas o todas las sustancias gustativas y la disgeusia es aquella en la que se presenta una distorsión en la percepción de un sabor ${ }^{(2)}$.

En el sabor no sólo interviene el gusto, sino se define como una interacción compleja de éste con el olfato y la sensibilidad somática.

El neuroepitelio olfatorio se localiza en la parte superior de ambas cavidades nasales. La conexión con el bulbo olfatorio se produce a través del nervio olfatorio. Además, las fibras nerviosas que llevan la información desde los receptores nasales hasta la corteza cerebral atraviesan áreas del cerebro que intervienen en las emociones y en la memoria. Por eso, muchos olores provocan reacciones físicas y emocionales. La mejor función olfatoria se da entre los 20 y los 40 años y a partir de ahí, va disminuyendo progresivamente.

Los trastornos del sentido del olfato son producidos por alteraciones que interfieren en el acceso de la sustancia que se ha olido al neuroepitelio olfatorio, lesiones de la región receptora (pérdida sensorial) o de las vías olfatorias centrales. Las causas más frecuentes de trastornos olfatorios son los traumatismos craneoencefálicos y las infecciones víricas ${ }^{(1)}$.

En muchas ocasiones pueden ir unidas alteraciones en la percepción del gusto y del olfato.

\section{DESARROLLO DEL TEMA}

Muchos fármacos pueden inducir trastornos del gusto $y / o$ del olfato y esto puede contribuir a que el paciente tenga menos apetito, no disfrute de la comida e, incluso, que pierda peso. En una sociedad como la nuestra en la que la comida forma parte de las relaciones sociales y se asocia al placer, puede contribuir a un deterioro importante de la percepción de calidad de vida de la persona. Se han descrito distintos problemas asociados en los pacientes con este tipo de alteraciones: para cocinar $(73 \%)$, cambios de humor $(68 \%)$, pérdidas de apetito (56\%) e insuficiente percepción de su propio olor corporal $(41 \%)^{(3)}$.

La incidencia de estos trastornos asociados a la quimioterapia alcanza hasta un $77 \%$ en los distintos estudios. Suelen comenzar al inicio de los tratamientos y en la mayoría de ellos desaparece tras su finalización aunque a veces persisten las alteraciones para el sabor salado durante más tiempo ${ }^{(3-5)}$.

En un estudio realizado en 87 pacientes con degustación de distintos sabores antes, durante y a los 3 meses de finalizado el tratamiento con quimioterapia, se objetivó que el $75 \%$ de los enfermos tenían alteraciones del olor y sabor de los alimentos durante el tratamiento pero que a los 3 meses volvían a ser iguales que antes de su inicio. En la mayoría, producía anorexia, pérdida de peso y una menor energía para poder llevar a cabo las actividades de la vida diaria( ${ }^{(3)}$. 
El mecanismo por el que los fármacos citostáticos causan trastornos del sentido del gusto no es del todo conocido ${ }^{(6,7)}$. La quimioterapia puede dañar directamente las papilas gustativas, causando alteraciones en la percepción que variarán según los pacientes; algunas como la ciclofosfamida y la vincristina, producen un sabor especial incluso inmediatamente después de inyectarlas. Otras causas se deben a que pueden afectar a la producción de saliva y mucosidad y, además, ser secretados en la saliva o a través del plasma en la cavidad oral ${ }^{(8)}$.

Muchos de los quimioterápicos producen con frecuencia alteraciones como los platinos, el fluoracilo, el metotrexate, la adriamiciana y los taxanos ${ }^{(2)}$. Se ha considerado como una de las toxicidades más estresantes para estos enfermos unida a la astenia, las naúseas, los vómitos y la pérdida de pelo ${ }^{(9,10)}$. Además, en la mayoría de los casos, los fármacos se suelen utilizar en esquemas de poliquimioterapia por lo que no es posible separar el efecto de una sola sustancia del de la interacción entre distintos compuestos ${ }^{(6)}$.

Hay ciertos tumores con una mayor prevalencia por el tipo de localización neoplásica y el tratamiento recibido que, a menudo combina radioterapia locorregional, como son los de cabeza y cuello pero se puede dar en muchos otros tipos fuera de esa región.

Los pacientes con tabaquismo importante y de edad avanzada suelen referir menos alteraciones del gusto por quimioterapia. Esto se debe a que presentan una hipogeusia de base y notan con menor intensidad los cambios que se producen cuando se administran los fármacos citostáticos. Respecto al olfato se ha descrito una mayor afectación en personas mayores por el número más reducido, una menor capacidad de regeneración y la mayor sensibilidad de los receptores olfatorios a las sustancias neurotóxicas como la quimioterapia ${ }^{(11,12)}$.
Los trastornos del gusto fueron incorporados en los criterios de toxicidad de la quimioterapia del Instituto Nacional del Cáncer en 1999. La escala de calidad de vida de la EORTC (European Organization for Research and Treatment of Cancer) QLQ-C30 es una herramienta internacional validada que comprende cinco tipos de escalas funcionales (física, social, emocional, cognitiva y personal), una escala global y nueve escalas de síntomas entre los que figuran las pérdida de apetito con dos ítems específicos que evalúan el sentido del gusto a través de dos preguntas: "¿Ha tenido problemas del sentido del gusto?" y "¿Le sabe diferente la comida y la bebida que usualmente?"16,13,14).

En un estudio reciente se investigó la relación de los trastornos del gusto y otros parámetros de calidad de vida en 197 pacientes con cáncer de pulmón, páncreas y colorrectal. Se objetivó un 70\% de pacientes con trastornos de la percepción del sabor y se asoció de forma significativa con distintas dimensiones de calidad de vida, especialmente la pérdida de apetito y la astenia. No hubo diferencias significativas entre los tres tipos de tumores en cuanto a incidencia de estas alteraciones aunque fueron más severas en los pacientes tratados de cáncer de colon. El régimen que lo produjo con mayor frecuencia e intensidad fue el irinotecán seguido de esquemas de combinación con oxaliplatino. Un $26 \%$ del global de los pacientes tuvieron una afectación leve del gusto y un 17,6\% moderada-severa $^{(6)}$.

En una revisión sistemática de los trabajos relacionados con las alteraciones del sabor y del olor asociadas al tratamiento citostático se incluyeron aquellos estudios que se centraban en pacientes adultos que sólo recibían quimioterapia como única modalidad de tratamiento oncológico específico y centrados en esta toxicidad. De los 163 trabajos revisados sólo cumplían los criterios 31 de ellos con niveles de evi- 
dencia del II al IV. Sus principales conclusiones señalaban que había una falta de consistencia de los resultados de los diferentes estudios como consecuencia de los diferentes diseños, el tipo de quimioterapia y el tipo y estadio tumoral. Se encontraron evidencias de la relación de los trastornos del gusto y del olfato con la quimioterapia y su asociación con la falta de apetito por la aversión que se produce a los alimentos, pero con reversibilidad de estos efectos una vez acabado el tratamiento ${ }^{(15)}$.

En cuanto al tipo de trastorno, en un estudio de caso-control se concluye que la disminución del gusto, la distorsión de éste $y$, sobre todo, el mal sabor de boca son los problemas más frecuentes ${ }^{(16)}$.

Puede haber aversión a un tipo de sabor o, o que es más común, de alimento en concreto. Una de las principales quejas es la distorsión del sabor de la carne, el plátano, las cremas o el café. La sensación más frecuente se describe como un "sabor metalizado" de determinadas comidas ${ }^{(17)}$.

A pesar de todo ello, las alteraciones en la percepción de los sabores inducidas por los tratamientos oncológicos específicos han sido poco valoradas por los especialistas implicados en el manejo de estos pacientes.

\section{Diagnóstico del problema}

Ante un paciente en tratamiento con quimioterapia hay que obtener una completa información clínica a través de una anamnesis que incluya preguntas específicas de la percepción de los sabores básicos.

En segundo lugar, conviene realizar una completa exploración de la boca con especial atención a la higiene bucal y posibles alteraciones odontológicas que pueden ser causa en si de las alteraciones del sentido del gusto o empeorarlas.

Las pruebas específicas de la evaluación de este trastorno son la realización de test de los cuatro sabores básicos con la utilización de cloruro sódico para el salado, sacarosa para el dulce, ácido cítrico para el ácido y café para el amargo. La evaluación debería hacerse en los dos lados de la boca.

Otra forma, mucho más complicada y menos factible, es la utilización de estímulos eléctricos débiles en la cuerda timpánica o el nervio glosofaríngeo (electrogustometría ${ }^{(2)}$. Hay algunos estudios con este método durante y después del tratamiento quimioterápico. Minakata et al. lo investigaron en una mujer con un cáncer de pulmón de células pequeñas que recibía cisplatino y etopósido. No había alteraciones en el nervio glosofaríngeo hasta el octavo día de la quimioterapia ${ }^{(18)}$. Ovensen y et al.estudiaron 26 pacientes con cáncer de pulmón, 5 con un tumor maligno de mama y 6 con carcinoma de ovario. Sólo en los casos de tratamientos para el cáncer de pulmón hubo cambios neurológicos significativos antes y durante la quimioterapia $^{(14)}$. Yamagata y et al. mostraron que, después de dos semanas de quimioterapia, casi todos los pacientes que no recibieron una infusión de zinc desarrollaron este trastorno ${ }^{(19)}$. Berteretche et al. midieron la actividad neurológica en los días 1 y 11 de quimioterapia encontrando diferencias significativas $^{(20)}$.

A pesar de todas estas opciones, en la práctica clínica para los trastornos del sentido del gusto en estos pacientes, suele bastar con una buena anamnesis y exploración de la cavidad bucal en busca de hallazgos que permitan mejorar el problema.

Para la cuantificación, se han utilizado distintos niveles de intensidad de las alteraciones como escalas analógicas visuales o cuestionarios subjetivos.

No hay pruebas específicas para explorar las alteraciones del sentido del olfato en este contexto y se suelen utilizar escalas que incluyen una puntuación acerca del 
olor percibido que va del $1=$ "me disgusta mucho" al 9= "me gusta mucho" (21).

\section{Intervenciones}

La literatura referente a las posibles intervenciones para mejorar, o, en el mejor de los casos, evitar este problema, es escasa y sin una sólida evidencia científica de su eficacia. Los pacientes que reciben un tratamiento antineoplásico deben ser informados de la posible toxicidad que puede tener en el ámbito de los sentido del gusto y del olfato para que el enfermo no se sienta desprevenido si aparece el efecto secundario y reforzar la idea de que es transitorio y se suele resolver en un breve espacio de tiempo una vez que finaliza el tratamiento. Los consejos dietéticos suelen ser de gran ayuda con la sustitución de las proteínas y los nutrientes de aquellos alimentos que provoquen las alteraciones para prevenir déficits. Se suele aconsejar evitar comidas con olores penetrantes.

Otras recomendaciones incluyen el mantener una correcta higiene bucal evitando la utilización de cepillos de dientes y colutorios abrasivos y masticar chicles sin azúcar o cubitos de hielo.

En el año 1999, Wickham et al. desarollaron distintas sugerencias para disminuir o mejorar los cambios de sabor de los alimentos con quimioterapia. Entre ellas se incluía el añadir más especias y condimentos a las comidas para darles un sabor más intenso, evitar la carne si producía un sabor metálico, evitar las comidas con mucho olor, evitar alimentos demasiado calientes, no comer con utensilios metálicos, beber una mayor cantidad de agua y cepillarse los dientes cuidadosamente después de cada ingesta ${ }^{(22)}$.

Además de todo esto, es importante el buen control de otros efectos secundarios digestivos como nauseas y los vómitos que pueden contribuir a unas peores consecuencias del problema.
Las deficiencias de zinc pueden inducir también trastornos del gusto. En estos casos, los suplementos con zinc a dosis entre 25 y $100 \mathrm{mg}$ pueden tener un efecto beneficioso $^{(2,23,24)}$.

A pesar de las sugerencias dadas, en un estudio Ilevado a cabo en Estados Unidos en 37 enfermos tratados con quimioterapia, sólo un 3\% de los pacientes habían seguido los consejos que previamente se habían dado para el manejo de los cambios del gusto. De éstos, sólo un 16\% reflejó que le habían ayudado mucho con respecto a este problema ${ }^{(25)}$.

\section{CONCLUSIONES}

Aunque no es uno de los efectos adversos de la quimioterapia más conocidos ni estudiados, las alteraciones del gusto y del olfato secundarias a ella son prevalentes en el grupo de los enfermos con cáncer. Se ha demostrado que producen un deterioro en su calidad de vida en un aspecto que se relaciona con las relaciones sociales y al que se da tanta importancia en nuestro medio, como es el de la comida.

En la mayoría de los estudios se concluye que se presenta en un alto porcentaje de pacientes pero se remarca su reversibilidad al poco tiempo de terminar los tratamientos.

Además, hay una falta de consistencia de los resultados de los diferentes trabajos como consecuencia de los diferentes diseños, la clase de quimioterapia y el tipo y estadio tumoral.

Mientras que se suele dar consejos e información detallada de otros efectos secundarios de los tratamientos empleados en oncología, una queja común es la falta de información acerca de éste para que el paciente esté prevenido y pueda enfrentarse a este problema de una mejor manera.

Es importante, desde el principio, dar recomendaciones dietéticas que incluyan las alteraciones de los sabores y olores que 
pueden producir estos tratamientos y preguntar al paciente por ellas en cada ciclo de quimioterapia. Para su cuantificación se han utilizado sobre todo cuestionarios subjetivos y escalas analógicas visuales. Las exploraciones específicas para su detección no se suelen realizar de rutina aunque si es conveniente descartar otros procesos y hacer una cuidadosa exploración bucal, buscando otros problemas que puedan empeorar este cuadro.

Por último, sería conveniente realizar más estudios acerca de este tema desde su fisiopatología hasta su influencia en la calidad de vida de los pacientes con cáncer así como el desarrollo de herramientas validadas para su valoración.

\section{REFERENCIAS BIBLIOGRÁFICAS}

1. Heckmann JG, Heckmann SM, Lang CJ, Hummel T. Neurological aspects of taste disorders. Arch Neurol 2003;60:66771.Doi:10.1001/archneur.60.5.667

2. Naik BS, Shetty N, Maben EVS. Druginduced taste disorders. Eur J Intern Med 2010; (21):240-3. Doi: 10.1016/j. ejim.2010.01.017

3. Steinbach S, Hummel T, Bohner C, Berktold S, Hundt W, Kriner M et al. Qualitative and quantitative assessment of taste and smell changes in patients undergoing chemotherapy for breast cancer or gynecologic malignancies. J Clin Oncol 2009;27(11):1899-905. Doi: 10.1200/ JCO.2008.19.2690

4. Grindel CG, Cahill CA, Walker M. Food intake of women with breast cancer during their first six month of chemotherapy. Oncol Nurs Forum 1989;16:401-7.

5. Holmes S. Food avoidance in patients undergoing cancer chemotherapy. Support Care Cancer 1993;1:326-30.

6. Zabernigg A, Gamper E, Giesinger JM, Rumppold G, Kemler G, Gattringer K et al. Taste alterations in cancer patients receiving chemotherapy: A neglected side effect? Oncologist 2010;15:913-20. Doi: 10.1634/theoncologist.2009-0333

7. Henkin RI. drug-induced taste and smell disorders. Incidence, mechanisms and management related primarily to treatment of sensory receptor dysfunction. Drug Saf 1994;11:318-77.

8. Corneau TB, Epstein JB, Migas C: Taste and smell dysfunction in patients receiving chemotherapy: A review of current knowledge. Support Care Cancer 2001; 9:575-80.

9. Bernhardson BM, Tishelman C, Rutqvist LE. Self-reported taste and smell changes during cancer chemotherapy. Support Care Cancer 2008;16:275-83. Doi:10.1007/ s00520-007-0319-7

10. Lindley C, McCune JS, Thomason TE, Lau$\operatorname{der}$ D, Sauls A, Adkins S et al. Perception of chemotherapy side effects cancer versus noncancer patients. Cancer Pract 1999; 7:59-65.

11. Doty RL, Shaman P, Applebaum SL, Giberson R, Siksorski L, Rosenberg L. Smell identificatiion abillity: Changes with age. Science 1984; 226:1441-3.

12. Rawson NE. Olfactory loss in aging. Sci Aging Knowledge Environ 2006; 5:6. Doi: 10.1126/sageke.2006.5.pe6

13. US. National Institutes of Health. CTEP Legacy CTC and CTCAE Versions. En línea [Acceso 11 de enero de 2013]. Disponible en: http://ctep.info.nih.gov/protocolDevelopment/electronic_applications.

14. Ovesen L, Sorensen M, Hannibal J. Electrical taste detection thresholds and chemical smell detection thresholds in patients with cancer. Cancer 1991; 68:2260-5.

15. Boltong A, Keast R. The influence of chemotherapy on taste perception and food hedonics: A systematic review. Cancer Treat Rev 2012; 38:152-63. Doi: 10.1016/j.ctrv.2011.04.008

16. Sánchez-Lara K, Sosa-Sánchez R, GreenRenner D, Rodríguez C, Laviano A, Motola-Kuba $D$, et al. Influence of taste disorders on dietary behaviors in cancer 
patients under chemotherapy. Nutr J 2010; 9:15-20. Doi: 10.1186/1475-2891-9-15

17. Boltong A, Keast R, Aranda S. Experiences and consequences of altered taste-flavour and food hedonics during chemotherapy treatment. Support Care Cancer 2012; Doi: 10.1007/s00520-012-1398-7.

18. Minakata Y, Yamagata T, Nakanishi H. Severe gustatory disorder caused by cisplatin and etoposide. Int J Clin Oncol 2002; 7:124-27.

19. Yamagata $T$, Nakamura $Y$, Yamagata $Y$, Nakanishi M, Matsunaga K, Nakanishi H et al. The pilot trial of the prevention of the increase in electrical taste thresholds by zinc containing fluid infusion during chemotherapy to treat primary lung cancer. J Exp Clin Cancer Res 2003; 22:557-63.

20. Berteretche MV, Dalix AM, d'Ornano AM, Bellisle F, Khayat D, Faurion A. Decreased taste sensitivity in cancer patients under chemotherapy. Support Care Cancer 2004; 12(8):571-6. Doi: 10.1007/s00520-0040589-2
21. Lawles HT, Sinopoli D, Chapman KW. A comparison of the labelled affective magnitude scale and the 9-point hedonic scale and examination of categorical behaviour. J Sens Stud 2010; 25:54-66. Doi: 10.1111/j.1745-459X.2010.00279.x

22. Wickham RS, Rehwaldt M, Kefer C, Shott S, Abbas K, Glynn-Tucker E et al. Taste changes experienced by patients receiving chemotherapy. Oncol Nurs Forum 1999; 26(4):697-706.

23. Briggs ER. Taste disturbances related to medication use. Consult Pharm 2009; 24(7):53843. Doi: 10.4140/TCP.n.2009.538

24. Doty RL, Shah M, Bromley SM. Druginduced taste disorders. Drug Saf 2008; 31(3):199-215. Doi: 10.2165/00002018200831030-00002

25. Rehwaldt M, Wickham R, Purf S, Tariman J, Blendowski C, Shott S, et al. Self-care strategies to cope with taste changes after chemotherapy. Oncol Nurs Forum 2009;36(2):47-56. Doi: 10.1188/09.ONF. E47-E56 
